\title{
Search for long-lived particles at LHC
}

\author{
Stefano Giagu ${ }^{1, a}$ \\ ${ }^{1}$ Sapienza Università di Roma and INFN Roma, Roma, IT
}

\begin{abstract}
Several scenarios beyond the Standard Model predict long-lived particles resulting in a wide variety of detector signatures depending on the nature of the particles and the decay lengths. Signals from long-lived particles are investigated by the ATLAS and CMS experiments exploiting different signatures, ranging from abnormal energy losses, to appearing or disappearing tracks, displaced vertices, lepton-jet signatures, long time-of-flight or late calorimetric energy deposits. This contribution summarizes the most recent results of the searches performed at the Large Hadron Collider (LHC) with the ATLAS and CMS detectors during the Run-1 data taking campaign. No evidence of any new physics is observed so far in any analysis, and the results are used to set stringent constraint on supersymmetric or hidden sector models.
\end{abstract}

\section{Introduction}

A variety of proposed theories beyond the Standard Model (BSM), such as supersymmetry (SUSY), theories with extra dimensions, scenarios with a hidden valley, and various others, can lead to the production of unusual signatures in detectors at the Large Hadron Collider (LHC). These may include: long-lived particles (LLP), meta-stable particles and collimated jets of displaced leptons or hadrons. Exploring these signatures is being of great interest today since they could give a possibility to evade the current constraints of SM extensions, tightened by the experimental evidence of the absence of any signal beyond Standard Model in the generic searches performed at LHC.

The ATLAS [1] and CMS [2] collaborations explored different experimental signatures and theoretical models using data collected from proton-proton collisions at LHC. This contribution gives an overview of some of the results of the searches performed on the whole LHC data taken at $\sqrt{s}=7$ and $8 \mathrm{TeV}$.

The following results are discussed here:

- gluinos being stable and decaying late in out of bunch crossings in ATLAS [3],

- heavy stable charged particles in ATLAS and CMS [4, 5],

- charginos with a significant lifetime giving rise to disappearing tracks in ATLAS [6],

- searches for long-lived neutral particles with a lifetime such that they decay within the ATLAS and CMS detector, but at a significant displacement from the primary event vertex (displaced supersymmetry) [4, 7-10],

- dark and hidden sectors inspired long-lived particles searches in ATLAS [11, 12], and CMS [8, 9].

\footnotetext{
a e-mail: stefano.giagu @ roma1.infn.it
}

\section{Supersymmetry inspired long-lived particles searches}

\subsection{Stable gluinos and stopped R-hadrons}

The Higgs boson measured at $125 \mathrm{GeV}[13,14]$ motivates heavy scalar scenarios where the squarks and sleptons are rendered heavy while gauginos may still be light (at the $\mathrm{TeV}$ scale or below) enough to be produced at the LHC energies. Split SUSY $[15,16]$ is a possibility that compromises with the current constraints. In such a scenario, gluinos decay via internal heavy squark lines, leading to their significantly long lifetimes and hadronic bound states (so-called $R$-hadrons). Massive $R$-hadrons could be detected as low- $\beta$ particles leaving activities due to large energy loss. Especially when they are produced nearly at the production threshold in $p p$ collisions at the LHC energies, they could come to rest inside the calorimeter and decay after a significant time, which leads to a unique signature of energetic jets delayed with respect to the collision timing. The ATLAS Stopped $R$-Hadrons analysis search for $R$-hadrons by looking for jets in "empty" bunch crossings, BCIDs with no protons in either beam. The search for such a signature is performed by using the full 7 and $8 \mathrm{TeV} p p$ collision data, and adopting a dedicated trigger that selects events containing energetic jets in empty bunch crossings of the LHC. Background events mimicking the signal signature predominantly originate from cosmic rays and upstream beam-halo interactions. Background from cosmic rays is effectively suppressed by vetoing events with reconstructed muon segments. Excluding events with spike-like signals in the calorimeter rejects background from random electronic noise and also reduces the contamination from cosmic rays and beam halo. Given the leading jet energy distribution for the candidate events, no excess above the background expectation is found and upper limit on the cross section of the pair-gluino produc- 
tion is set as a function of the gluino mass for a given range of lifetime values, as shown in Fig. 1. Depending on model parameters, limits excluding $m_{\tilde{g}}<545-832$ GeVand $m_{\tilde{q}}<344-397 \mathrm{GeV}$ can be set.

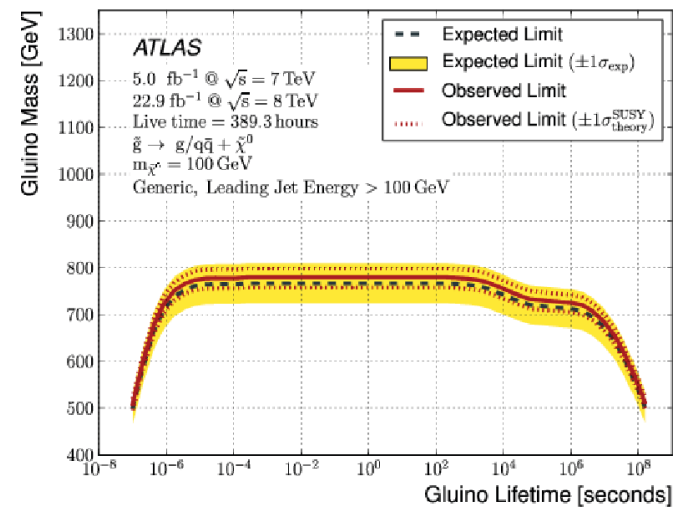

Figure 1. Limits on gluino mass versus its lifetime with $R$ hadron lifetimes in the plateau acceptance region between $10^{-5}$ and $10^{3}$ seconds.

\subsection{Heavy stable charged particles}

Heavy stable charged particles, traversing the ATLAS and CMS detectors, are identified by their velocities significantly lower than the speed of light and resulting large ionization losses.

The ATLAS search utilizes the $\beta$ measurements based on the time-of-flight measured in the muon spectrometer and calorimeters. Resolution on $\beta$ is optimized using large calibration data sample of muons from $Z$ decays. The reconstructed mass is finally obtained by averaging the two $\beta$ measurements in different detector elements. Background tracks dominantly originate from muons with mismeasured $\beta$; the mass spectrum of these tracks are estimated using a convolution of momentum and $\beta$ distributions of the signal search sample, relying on the fact that these two kinematic variables are fully uncorrelated for the background tracks.

The observed mass spectrum from ATLAS is shown in Figure 2(a) in which the candidate events are required to have two tracks with $p_{\mathrm{T}}>50 \mathrm{GeV}$ and $0.2<\beta<0.95$; no significant excess above the background expectation is found. In the same figure also the expected distribution from signals from staus predicted in gauge-mediated SUSY breaking (GMSB) models is also shown. Assuming that the stau is stable upper limits on the cross section of $\sim 1 \mathrm{fb}$ at $95 \% \mathrm{CL}$ in the stau mass range from 250 to $500 \mathrm{GeV}$ are obtained. These results can be interpreted in the context of gauge-mediated SUSY breaking [17] scenarios where a slepton is the next-to-lightest superpartner with a significantly long lifetime, as shown in Fig. 2(b).

The CMS experiment analysis is performed in five separate channels, to account for the wide variety of types of HSCP:

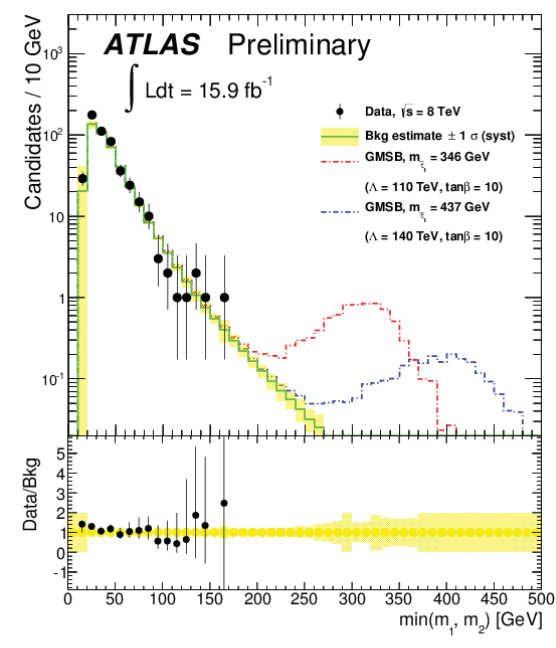

(a) The reconstructed mass distribution

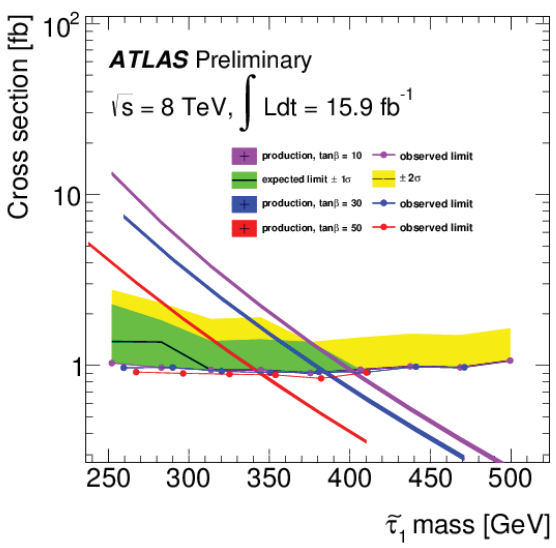

(b) Limits on the production cross section in GMSB scenarios

Figure 2. The reconstructed mass distribution(a) and limits on the production cross section as a function of stau mass in GMSB scenarios(b).

- the "tracker+TOF" analysis searches for singly-charged HSCPs requiring both a track in the inner tracker and in the muon system.

- the "tracker-only" analysis searches for singly-charged HSCPs requiring only a track in the inner tracker, to account for the possibility of material interactions causing the HSCPs to become neutral in their passage through the detector.

- the "muon-only" analysis searches for singly-charged HSCPs requiring only a track in the muon system.

- the "fractionally-charged" analysis searches for HSCPs with $|Q|<1 e$, requiring a track in the inner tracker with a $\mathrm{d} E / \mathrm{dx}$ smaller than that for a SM particle.

- the "multiply-charged" analysis searches for HSCPs with $|Q|>1 e$, requiring tracks in the inner tracker and muon system with a much higher $\mathrm{d} E / \mathrm{dx}$ than for the singly-charged analysis. 
In all cases, the observations agree with the expected background, so limits are set for different signal models, as shown in Figure 3.
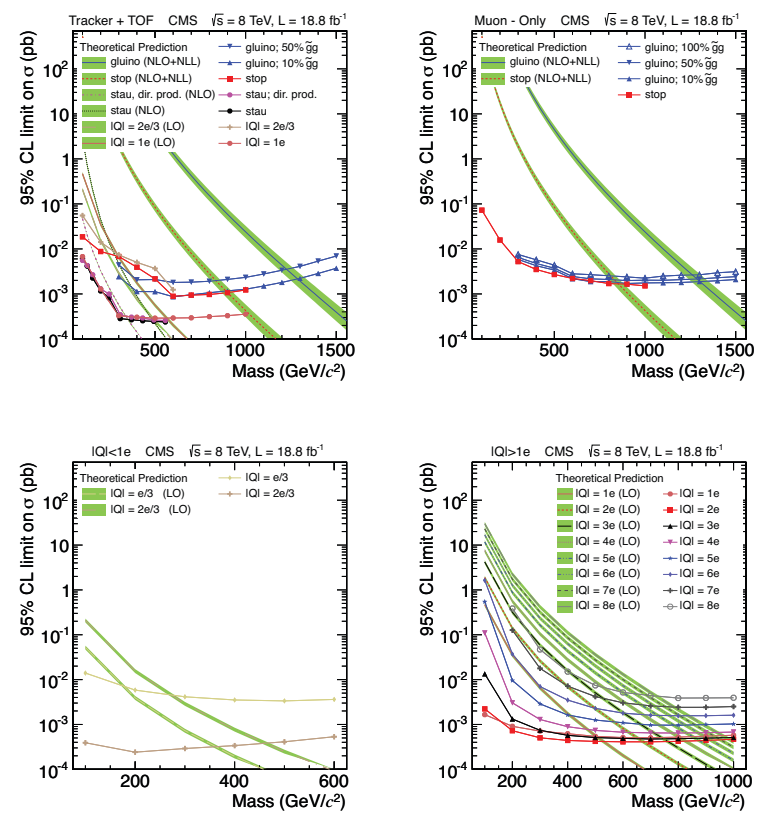

Figure 3. Limits for four of the HSCP analysis channels. Top from left to right: tracker+TOF, muon-only. Bottom from left to right: fractionally-charged, and multiply-charged. The different lines correspond to the different signal models for which the limits are set.

The obtained result can be reinterpreted in the context of different signal models [18], by constructing efficiency maps for reconstructing the long-lived particles as a function of $\beta$ and $\eta$ in bins of $p_{\mathrm{T}}$. Figure 4 shows an example of the application of this technique used to show points in the parameter space of the phenomenological minimal supersymmetric model (pMSSM) that can be excluded by this analysis.

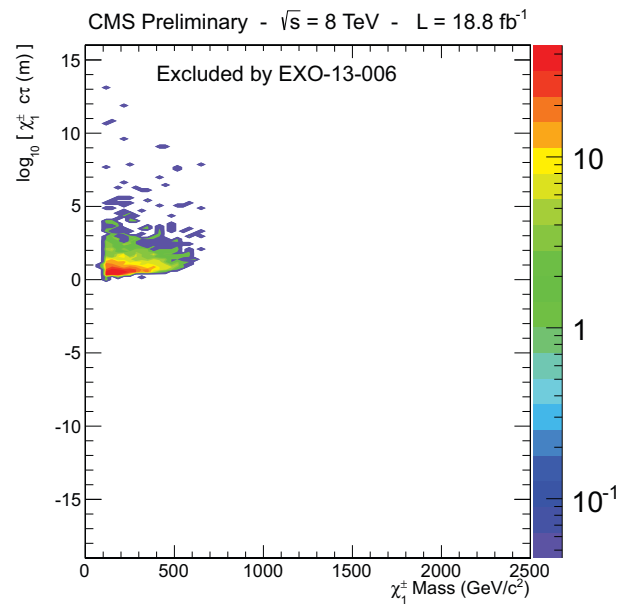

Figure 4. Parameter points in the pMSSM that can be excluded by reinterpretation of the HSCP search as described in the text.

\subsection{Disappearing tracks}

The ATLAS Disappearing Tracks analysis considers Anomaly Mediated SUSY Breaking (AMSB) [19, 20] models with almost a mass degenerate lightest chargino and neutralino. This can occur in scenarios where they are dominantly wino or higgsino, that are of interest as these retain a good candidate for dark matter, and could in principle accommodate the measured Higgs mass. One interesting feature of these scenarios is that the chargino has a considerable lifetime and predominantly decays into a neutralino plus a low-momentum charged pion. The lowmomentum charged pion track is rarely reconstructed due to its large displacement and a small number of interactions in the tracking system, therefore, a decaying chargino is typically recognized as a "disappearing track" that has few associated hits in the outer tracking volume. These signatures are identified in the ATLAS inner detector by searching for isolated, high $p_{\mathrm{T}}$ tracks that have well measured hits in the inner silicon trackers, but have a low number of hits in the outer straw tracker. Background in the signal region mostly comprises lower $p_{\mathrm{T}}$ tracks that have been mis-measured, with non-identified muons also contributing.

The results of the ATLAS search for events with disappearing-track signature is shown in Figure 5, where the $p_{\mathrm{T}}$ spectrum of candidates tracks is compared to the expectation for the background and for signals corresponding to various choices of the chargino mass and lifetime. In the absence of a signal, constraints are set on the parameter space of the minimal AMSB model. In the context of the decoupled AMSB a lower limit on the lightest chargino mass of $m_{\tilde{\chi}_{1}^{ \pm}}>270 \mathrm{GeV}$ at $95 \% \mathrm{CL}$ is set, that directly constrains the wino dark matter mass.

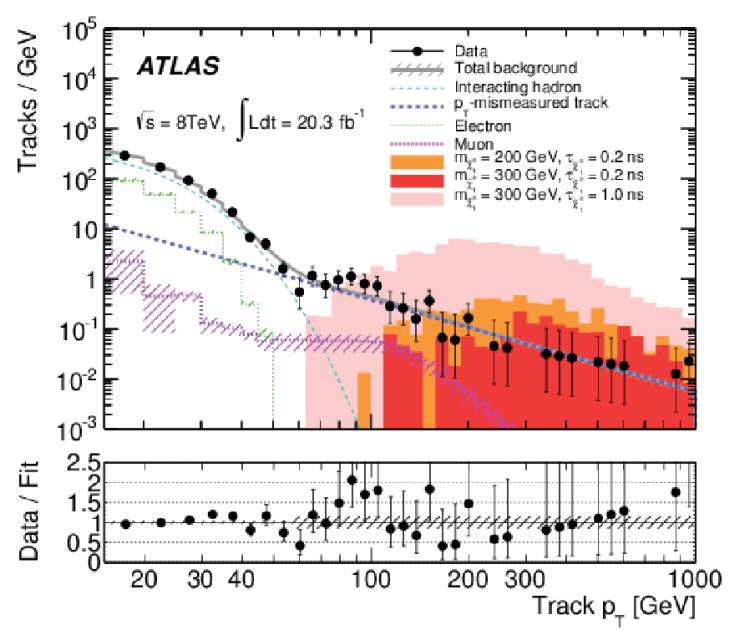

Figure 5. The $p_{\mathrm{T}}$ spectrum of disappearing-track candidates in the disappearing-track signature analysis performed by the ATLAS experiment. 


\subsection{Search for displaced Supersymmetry}

A specific search technique is used by the CMS experiment to explore a region of parameter space not covered by previous searches. In this tecnique a SUSY model where long-lived stop quarks are pair-produced and then undergo an $R$-parity violating decay to a $b$ quark and a lepton $(\tilde{t} \rightarrow b \ell)$ is considered. The analysis then searches for events in which one of the leptons is an electron and the other a muon, thus substantially reducing the background from SM processes. The leptons are required to be displaced, but they are not required to form a vertex, allowing the search to remain sensitive to a variety of new physics scenarios.

Signal candidate events are identified by looking for an event with a muon and an electron of opposite charge, both of which are required to be isolated and have $p_{\mathrm{T}}>$ $25 \mathrm{GeV}$. The leptons are required to have a transverse impact parameter $d_{0}$ less than $2 \mathrm{~cm}$, so that the tracking efficiency for the leptons remains high. Background from QCD HF events in the signal region is obtained using data driven methods, while background from other sources is estimated from simulation. In all search regions studied by CMS, the observations agree with the expected background, so limits are set on the stop quark mass as a function of lifetime as shown in Figure 6.

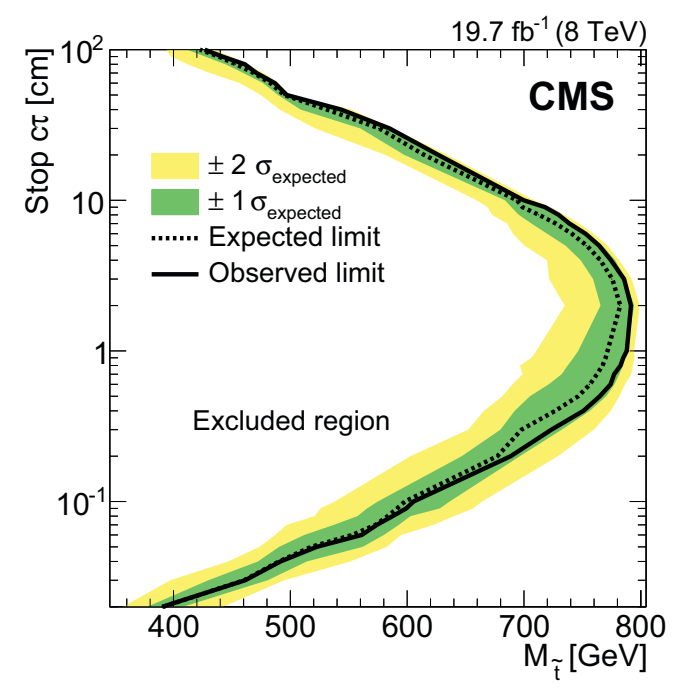

Figure 6. Expected and observed 95\% CL cross section exclusion contours for top squark pair production in the plane of top squark lifetime $(c \tau)$ and top squark mass. These limits assume a branching fraction of $100 \%$ through the RPV vertex $\tilde{t} \rightarrow b \ell$, where the branching fraction to any lepton flavor is equal to $1 / 3$. As indicated in the plot, the region to the left of the contours is excluded by this search.

The ATLAS experiment search for signals from $R$ parity violating displaced supersymmetry by exploiting an enhanced track reconstruction to efficiently identify displaced vertices originating from heavy decaying particles with the lifetime range from $O\left(10^{-12}\right)$ to $O\left(10^{-9}\right)$ seconds. The present search is performed using events with a multitrack displaced vertex that contains a high- $p_{\mathrm{T}}$ muon, and its result is interpreted in the context of a $R$-parity violating scenario in which non-zero value of $\lambda_{2 i j}^{\prime}$ allows the lightest neutralino to decay into a muon and jets. Candidate events are selected with the requirements of a high- $p_{\mathrm{T}}$ muon and a displaced vertex. In order to suppress background events containing displaced vertices originating from secondary interactions, only vertices located outside the material of the inner detector are considered. The vertex is finally required to have a mass $\left(m_{\mathrm{DV}}\right)$ above $10 \mathrm{GeV}$ and a charged multiplicity $\left(N_{\text {trk }}\right)$ larger than 4 . No event is found in the signal region while the expected number of background events (predominantly originating from random combinations of tracks and hadron interactions with air molecules) is $0.02 \pm 0.02$. This translate in an upper limit of 0.14 $\mathrm{fb}$ is set on the visible cross section at $95 \% \mathrm{CL}$. The corresponding 95\% CL upper limits on the production cross section is also set for some squark and neutralino masses as a function of the neutralino lifetime, as shown in Fig. 7.

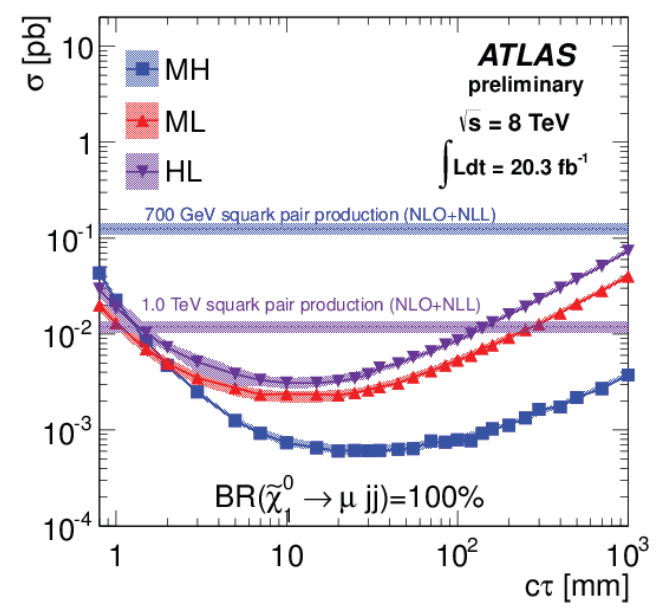

Figure 7. Upper limit on the squark-pair production cross section as a function of the neutralino lifetime for different combinations of squark and neutralino masses. The labels in the plot indicate the squark neutralino masses in GeV: 700-494 (MH), 700-108 (ML), and 1000-108 (HL). The branching ratio of the decay chain from squark to neutralino to muon-plus-jets is set to $100 \%$.

\section{Dark and Hidden sectors inspired Long-Lived Particles searches}

Several possible extensions of the Standard Model predict the existence of a hidden sector that is weakly coupled to the visible one (e.g. refs. [21-26]). Depending on the structure of the hidden sector and its coupling to the SM, some unstable hidden states may be produced at colliders and decay back to SM particles with sizeable branching fractions. For example, in supersymmetric theories, the lightest visible super-partner may decay into hidden particles, some of which can decay back to the visible sector (see e.g. refs. [22, 26, 27]). Several other distinct, nonsupersymmetric, examples exist (see e.g. refs. [21, 2325]). If the lightest unstable hidden states have masses 
in the $\mathrm{MeV}$ to $\mathrm{GeV}$ range, they would decay mainly to leptons and possibly light mesons. A particle from the hidden sector can be produced in certain weak interaction decays, such as that of the Higgs boson, with the behavior of the produced particle determining the signature. If the particle itself couples weakly to the SM then it may travel some distance before decaying into SM particles. This makes the particle a neutral long-lived particle and can give rise to displaced vertex signatures in the detector. The particle may also produce a shower in the hidden sector. If dark photons are produced in these showers and couple to the SM, they may decay into highly collimated pairs of light SM particles, specifically electrons, muons or hadrons. Multiple dark photons may be radiated by the same hidden sector particle, with the resulting overlapping ones leading to jets with multiple pairs of particles. Decays of dark photons to heavier particle pairs, such as protons, are kinematically forbidden. Highly collimated jets of leptons do not occur in the SM, and are the subject of BSM searches. The dark photon may be long-lived, which can result in jets of lepton pairs originating from displaced vertices.

\subsection{Hidden Valley displaced decays}

The CMS experiment performed a search for displaced lepton sensitive to a wide class of models that contain long-lived particles decaying to leptons. For illustration purposes a specific signal model is described here: a nonStandard Model (SM) Higgs decays to a long-lived spinless boson $\mathrm{X}\left(H^{0} \rightarrow \mathrm{XX}\right)$, which then decays into a pair of leptons (either $\mathrm{X} \rightarrow$ ee or $\mathrm{X} \rightarrow \mu \mu$ ). Although in this model the long-lived particles are pair-produced and so we would expect to observe up to two displaced vertices per event, for maximum generality this search only requires one displaced vertex to be found.

The data is collected using a pair of triggers, one for each channel, requiring either two high-energy deposits in the electromagnetic calorimeter or two high-momentum tracks in the muon detector. In both cases, the tracker information is not used in the trigger, as the track reconstruction at the CMS high-level trigger (HLT) is not necessarily efficient for displaced tracks. In the muon case, the muons are reconstructed without any primary vertex constraint, and muons from cosmic rays are vetoed by requiring that the three-dimensional angle between the two muons be less than 2.5 radians.

Long-lived particle candidates are identified by looking for two isolated, energetic electrons (with $E_{T}>40$ $\mathrm{GeV}$ ) or muons (with $p_{\mathrm{T}}>26 \mathrm{GeV}$ ) that form a good vertex, and are required to be significantly displaced from the primary vertex (the significance of the transverse impact parameter $\left.\left|d_{0}\right| / \sigma_{d}>12\right)$. The background is estimated using a data-driven method, exploiting the fact that the angle $\Delta \phi$ between the flight direction to the secondary vertex and the dilepton momentum should be close to 0 for a true long-lived particle event, but randomly distributed for background. The estimated background is zero events, and zero events are observed, so limits are set. Figure 8 shows some sample limits obtained in the $H^{0} \rightarrow \mathrm{XX}$ model for a variety of different $m_{X}$ values and lifetimes.

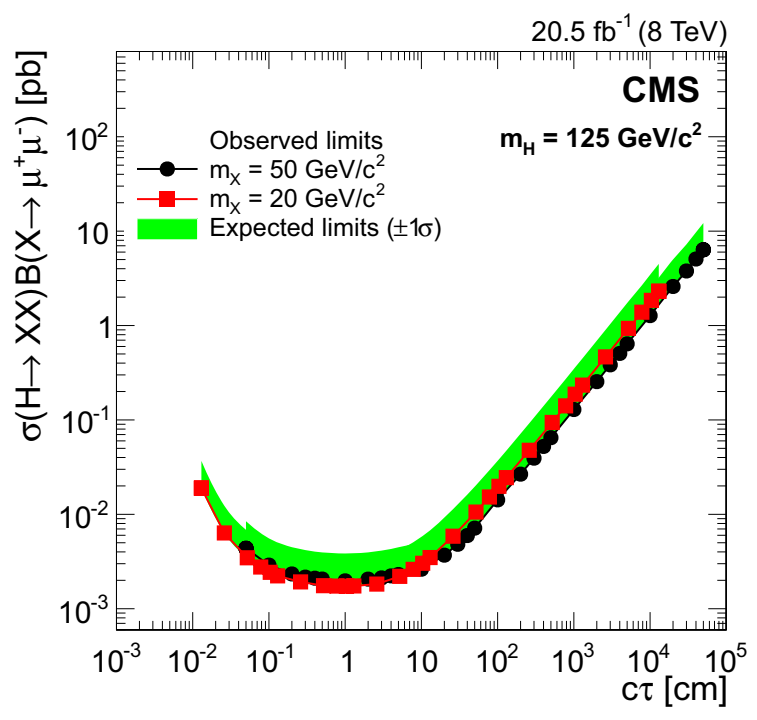

Figure 8. The $95 \% \mathrm{CL}$ upper limits on $\sigma\left(\mathrm{H}^{0} \rightarrow \mathrm{XX}\right) \mathrm{B}(X \rightarrow$ $\mu \mu$ ) for Higgs boson masses of $125 \mathrm{GeV}$ as a function of the $\mathrm{X}$ lifetime. Results are shown for several different $\mathrm{X}$ boson masses. Shaded bands show the $\pm 1 \sigma$ range of variation of the expected 95\% CL limits.

CMS searched also for displaced jets predicted in a similar way as done for the displaced leptons analysis, to cover the case where the long-lived neutral particle decays hadronically. In this case for the $H^{0} \rightarrow \mathrm{XX}$ model, the X bosons has been chosen to decay via $\mathrm{X} \rightarrow q q$.

Candidate events are selected by searching for two displaced jets with momentum $>60 \mathrm{GeV}$. In addition, requirements are placed on the number of prompt tracks and fraction of energy carried by prompt tracks, where a prompt track here is defined as a track with a transverse impact parameter less than $500 \mu \mathrm{m}$. The jets are then required to form a good-quality vertex. Finally, the tracks in the jet are hierarchically clustered and a likelihood discriminant is built for the jets based on the secondary vertex track multiplicity, the cluster root-mean-square (RMS), and the fraction of the secondary vertex tracks having a positive value of the impact parameter. The selection criteria are optimized separately for models with lower decay lengths $\left(L_{x y}<20 \mathrm{~cm}\right)$ and higher $(>20 \mathrm{~cm})$. In the final results, two events are observed passing the low $L_{x y}$ selection and one passing the high $L_{x y}$ selection, both consistent with the expected background. Limits are thus set on the signal models; Figure 9 shows a sample of the limits for one of the studied cases.

The ATLAS experiment uses a specialist trigger to search for long-lived neutral particles predicted by Hidden Valley models decaying in jets inside the ATLAS hadron calorimeter. The results are interpreted in the context of an $\mathrm{HV}$ model with a $\pi_{v}$ long-lived particle $[21,25]$. The specialist trigger called "CalRatio trigger" is tuned for narrow jets with anomalous energy deposition in the hadronic calorimeter $\left(E_{H}\right)$ compared with the deposit in the elec- 


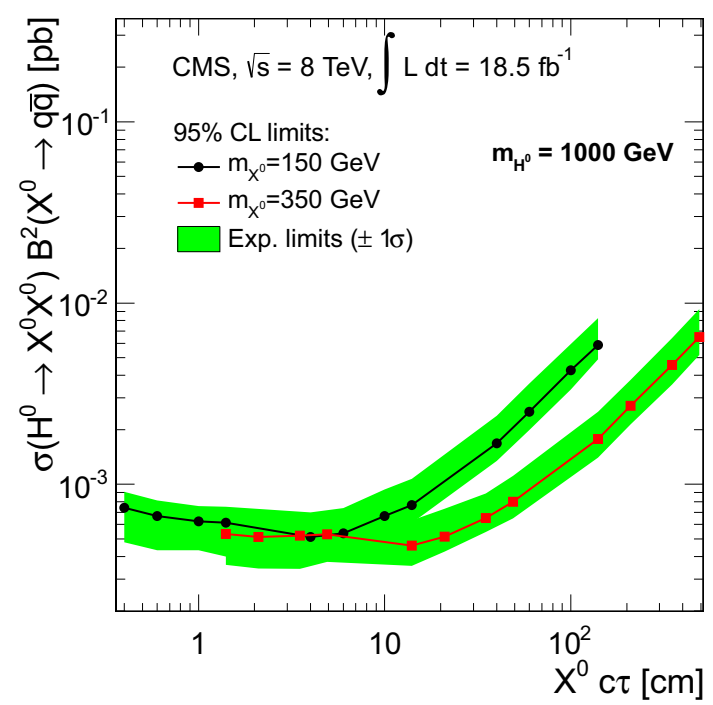

Figure 9. The $95 \% \mathrm{CL}$ upper limits on $\sigma\left(\mathrm{H}^{0} \rightarrow \mathrm{XX}\right) \mathrm{B}^{2}(X \rightarrow$ $q q$ ) for Higgs boson masses of $1000 \mathrm{GeV}$ as a function of the $\mathrm{X}$ lifetime. Results are shown for two different $\mathrm{X}$ boson masses. Shaded bands show the $\pm 1 \sigma$ range of variation of the expected 95\% CL limits.

tromagnetic calorimeter $\left(E_{E M}\right)$, and no associated tracks in the inner tracking detector. The analysis itself then reenforces these trigger conditions by requiring two jets with $\log _{10}\left(E_{H} / E_{E M}\right)>1.2$ and no good associated charged tracks with $p_{\mathrm{T}}>1 \mathrm{GeV}$. Figure 10 shows the jet energy distribution ratio between the hadronic calorimeter and the electromagnetic calorimeter for SM jets in data and MC signal samples and for a possible Hidden Valley signal. Using the full statistic collected at $\sqrt{s}=8 \mathrm{TeV}$, ATLAS excludes at $95 \% \mathrm{CL}$ decay lengths $0.37 \mathrm{~m}<c \tau_{\pi_{v}}<5.12 \mathrm{~m}$ for $m_{\pi_{v}}=25 \mathrm{GeV}$ and $m_{h}=126 \mathrm{GeV}$ [11].

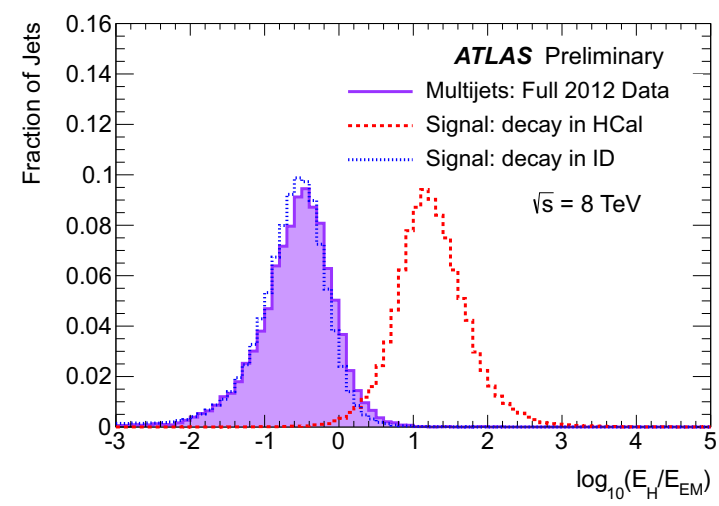

Figure 10. Jet energy distribution ratio between the hadronic calorimeter and the electromagnetic calorimeter for SM jets in data and MC signal samples and for a possible Hidden Valley signal.

\subsection{Search for displaced Dark photons decays}

An extensively studied case is one in which the SM and the hidden sectors couple via the vector portal, in which a light hidden photon (dark photon, $\gamma_{\mathrm{d}}$ ) mixes kinetically with the SM photon. If the hidden photon is the lightest state in the hidden sector, it decays back to SM particles with branching fractions that depend on its mass [26, 28, 29]. For the case in which the $\gamma_{\mathrm{d}}$ kinetically mixes with hypercharge, one finds that $\epsilon$, the kinetic mixing parameter, controls both the $\gamma_{\mathrm{d}}$ decay branching fractions and lifetime. More generally, however, the branching fractions and lifetime are model-dependent and may depend on additional parameters.

Due to their small mass, these particles are typically produced with a large boost and, due to their weak interactions, can have non-negligible lifetime. As a result one may expect, from dark photon decays, collimated jetlike structures containing pairs of electrons and/or muons and/or charged pions ("lepton jets", LJs) that can be produced far from the primary interaction vertex of the event (displaced LJs).

Neutral particles which decay far from the interaction point into collimated final states represent a challenge both for the trigger and for the reconstruction capabilities of the LHC detectors. Collimated charged particles in the final state can be difficult to disentangle due to the limited granularity of the detector. Moreover, in the absence of information from the inner tracking system, it is necessary to use the muon spectrometer (MS) for the reconstruction of tracks which originate from a secondary decay far from the primary interaction vertex (IP).

The high-resolution, high-granularity measurement capability of the ATLAS "air-core" MS is ideal for this type of search. In addition, the ATLAS inner tracking system can be used to define isolation criteria to significantly reduce, for decay vertices far from the interaction point, the otherwise overwhelming SM background from proton-proton collisions.

The results of the ATLAS search [12] performed on the complete dataset collected during the 2012 run at $8 \mathrm{TeV}$, for LJ production are used to set upper limits on the Higgs boson decay branching fraction to LJs as a function of the $\gamma_{\mathrm{d}}$ mean lifetime, according to the FRVZ models. The resulting exclusion limits on the $\sigma \times \mathrm{BR}$, assuming the Higgs boson $\mathrm{SM}$ gluon fusion production cross section $\sigma_{\mathrm{SM}}=$ $19.2 \mathrm{pb}$, are shown in figure 11 as a function of the $\gamma_{\mathrm{d}}$ mean lifetime (expressed as $\mathrm{c} \tau$ ) for two benchmark models in which the SM Higgs decay in two and four dark photons.

These results are also interpreted in the context of the Vector portal model as exclusion contours in the kinetic mixing parameter $\epsilon$ vs $\gamma_{\mathrm{d}}$ mass plane [30, 31] as shown in figure 12. Assuming Higgs decay branching fractions into $\gamma_{\mathrm{d}}$ of $5 / 10 / 20 / 40 \%$ and the NNLO gluon fusion Higgs production cross section, the lifetime limits can be converted into kinetic mixing parameter $\epsilon$ limits. While the other limits are model-independent because they produce the hidden photon through the vector portal coupling, this limit does depend on the additional 

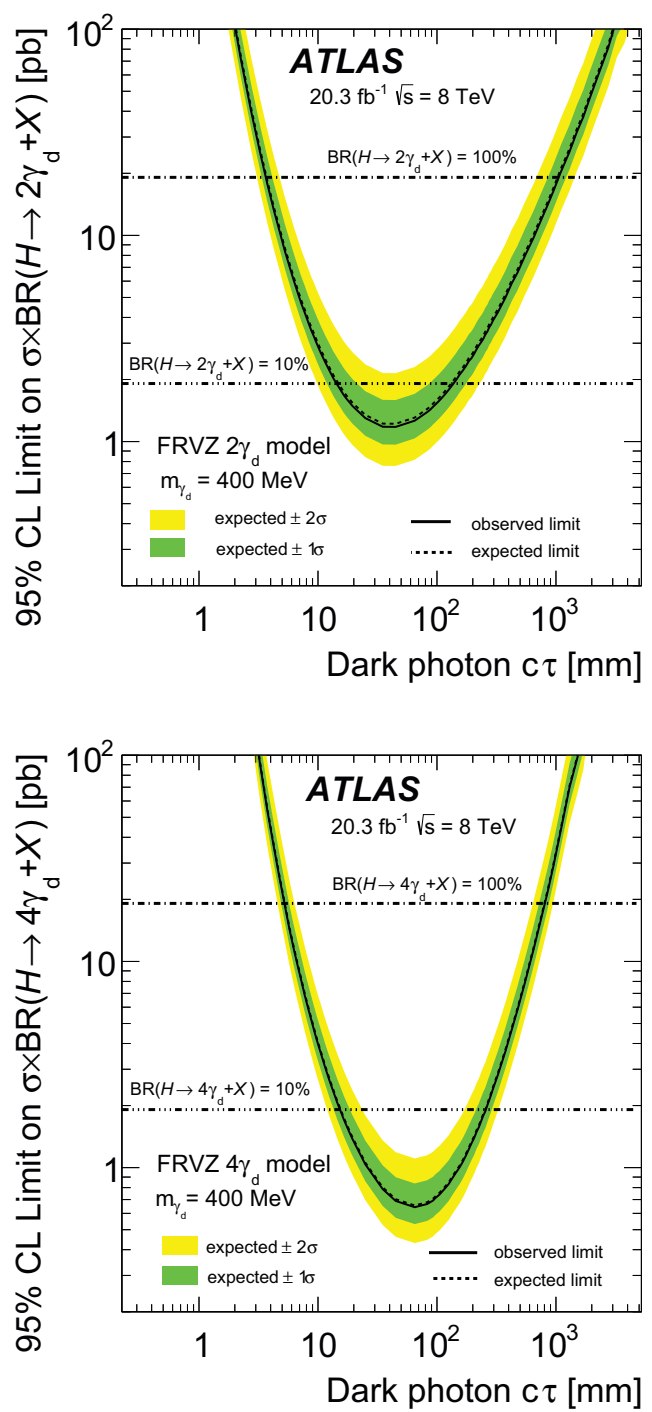

Figure 11. he $95 \%$ upper limits on the $\sigma \times \mathrm{BR}$ for the processes $H \rightarrow 2 \gamma_{\mathrm{d}}+X$ (left) and $H \rightarrow 4 \gamma_{\mathrm{d}}+X$ (right), as a function of the $\gamma_{\mathrm{d}}$ lifetime $(\mathrm{c} \tau)$ for the FRVZ benchmark samples. The expected limit is shown as the dashed curve and the almost identical solid curve shows the observed limit. The horizontal lines correspond to $\sigma \times \mathrm{BR}$ for two values of the $\mathrm{BR}$ of the Higgs boson decay to dark photons.

assumption on the Higgs branching fraction to the hidden sector. The resulting $90 \% \mathrm{CL}$ exclusion regions for $H \rightarrow 2 \gamma_{\mathrm{d}}+X$ are shown in figure 12 ; the $\gamma_{\mathrm{d}}$ mass interval (0.25-1.5) GeVcorresponds to the values in which the $\gamma_{\mathrm{d}}$ decay branching fractions and the detection efficiencies are comparable with those for the $0.4 \mathrm{GeV} \gamma_{\mathrm{d}}$ mass. The systematic uncertainties due to the detection efficiency and decay branching fraction variations as a function of the $\gamma_{\mathrm{d}}$ mass were estimated and included in the $90 \%$ CL exclusion region evaluations.

\section{Conclusions}

Long-lived particle and leptonic jet signatures, if observed, would be a clear indication of new physics beyond the

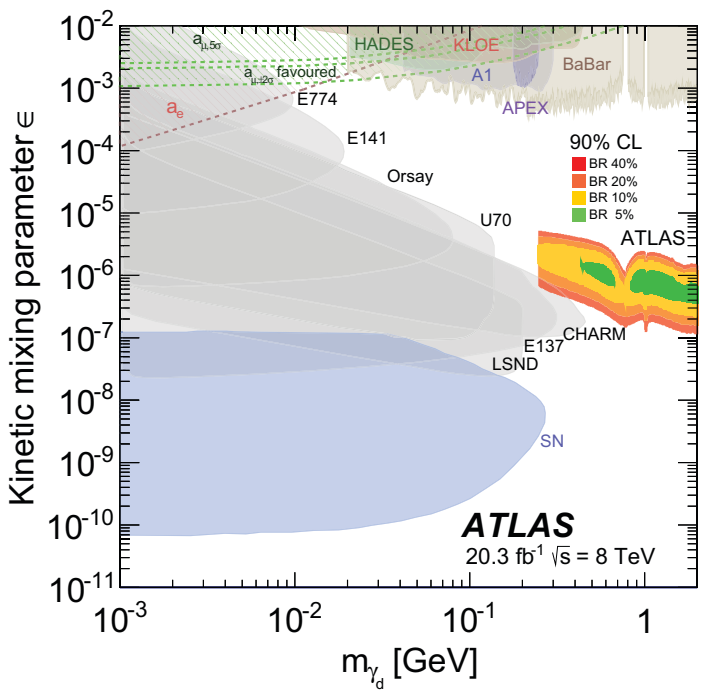

Figure 12. Parameter space exclusion plot for dark photons as a function of the $\gamma_{\mathrm{d}}$ mass and of the kinetic mixing parameter $\epsilon$. The 90\% CL exclusion limits from the ATLAS search, assuming the FRVZ model $H \rightarrow 2 \gamma_{\mathrm{d}}+X$ with decay branching fraction to $\gamma_{\mathrm{d}}$ of $5 / 10 / 20 / 40 \%$ and the NNLO gluon fusion Higgs production cross section, are shown.

Standard Model. The ATLAS and CMS experiments have performed a systematic program of searches for these signatures using the whole LHC Run-1 data, without finding any hint of signals from new physics to date. The next start of the Run-2 of LHC represents new potential for discovery due to the increased energy and luminosity, that in conjunction with novel sophisticated analysis techniques and detector and reconstruction improvements under development in both ATLAS and CMS may open avenues to search for signatures of new physics due to long-lived particles.

\section{References}

[1] ATLAS Collaboration, JINST 3 (2008) S08003.

[2] CMS Collaboration, JINST 3 (2008) S08004.

[3] ATLAS Collaboration, Phys. Rev. D 88, no. 11, 112003 (2013) [arXiv:1310.6584 [hep-ex]].

[4] ATLAS collaboration, ATLAS-CONF-2013-058, http://cdsweb.cern.ch/record/1595755.

[5] CMS Collaboration, JHEP 1307 (2013) 122, [arXiv:1305.0491 [hep-ex]].

[6] ATLAS Collaboration, Phys. Rev. D 88, no. 11, 112006 (2013) [arXiv:1310.3675 [hep-ex]].

[7] ATLAS collaboration, ATLAS-CONF-2013-092, http://cdsweb.cern.ch/record/1557775.

[8] CMS Collaboration, CMS-PAS-EXO-12-037, https://cds.cern.ch/record/1669814.

[9] CMS Collaboration, CMS-PAS-EXO-12-038, https://cds.cern.ch/record/1563591.

[10] CMS Collaboration, Submitted to PRL, [arXiv:1409.4789 [hep-ex]]. 
[11] ATLAS Collaboration, ATLAS-CONF-2014-041, https://cds.cern.ch/record/1740972.

[12] ATLAS Collaboration, JHEP11 (2014) 088, [arXiv:1409.0746 [hep-ex]].

[13] ATLAS Collaboration, Phys. Lett. B 716, 1 (2012), [arXiv:1207.7214 [hep-ex]].

[14] CMS Collaboration, Phys. Lett. B 716, 30 (2012), [arXiv:1207.7235 [hep-ex]].

[15] N. Arkani-Hamed and S. Dimopoulos, JHEP 0506, 073 (2005), [hep-th/0405159].

[16] N. Arkani-Hamed, S. Dimopoulos, G. F. Giudice and A. Romanino, Nucl. Phys. B 709, 3 (2005), [hepph/0409232].

[17] G. F. Giudice and R. Rattazzi, Phys. Rept. 322, 419 (1999), [hep-ph/9801271].

[18] CMS Collaboration, CMS Physics Analysis Summary CMS-PAS-EXO-13-006, https://cds.cern.ch/record/1648902

[19] G. F. Giudice, M. A. Luty, H. Murayama and R. Rattazzi, JHEP 9812, 027 (1998), [hep-ph/9810442 [hep$\mathrm{ph}]]$.

[20] L. Randall and R. Sundrum, Nucl. Phys. B 557, 79 (1999), [hep-th/9810155].
[21] M. J. Strassler and K. M. Zurek, Phys. Lett. bf B 651 (2007) 374, [arXiv:0604261 [hep-ph]].

[22] N. Arkani-Hamed and N. Weiner, JHEP 12 (2008) 104, [arXiv:0810.0714 [hep-ph]].

[23] T. Han, Z. Si, K. M. Zurek, and M. J. Strassler, JHEP 07 (2008) 008, [arXiv:0712.2041 [hep-ph]].

[24] S. Gopalakrishna, S. Jung, and J. D. Wells, Phys. Rev. D 78 (2008) 055002, [arXiv:0801.3456 [hep-ph]].

[25] M. J. Strassler and K. M. Zurek, Phys. Lett. B 661 (2008) 263-267, [arXiv:0605193 [hep-ph]].

[26] A. Falkowski, J. T. Ruderman, T. Volansky and J. Zupan, JHEP 05 (2010) 077, [arXiv:1002.2952 [hep-ph]].

[27] C. Cheung, J. T. Ruderman, L. Wang, I. Yavin, Phys. Rev. D 80 (2009) 035008, [arXiv:0902.3246 [hep-ph]].

[28] P. Meade, M. Papucci and T. Volansky JHEP 12 (2009) 052, [arXiv:0901.2925 [hep-ph]].

[29] B. Batell, M. Pospelov and A. Ritz, Phys. Rev. D 79 (2009) 115008, [arXiv:0903.0363 [hep-ph]].

[30] R. Essig et al., [arXiv:1311.0029 [hep-ph]].

[31] J. D. Bjorken, R. Essig, P. Schuster and N. Toro, Phys. Rev. D 80075018 (2009), [arXiv:0906.0580 [hep-ph]]. 